\title{
Application of distributed photovoltaic power generation technology in electric vehicles
}

\author{
Min $\mathrm{Wu}^{\mathrm{a}}$ \\ Tibet Autonomous Region Energy Research Demonstration Center, Lasa 850000, China
}

\begin{abstract}
In this paper, a photovoltaic grid connected electric vehicle charging station suitable for plateau application is developed by using two-way energy storage photovoltaic grid connected power generation technology; Introduce a medium-sized electric bus to carry out long-term operation test in Tibet; The detection experimental device and evaluation method suitable for electric vehicle power battery in plateau area are constructed; Carry out strategic research on the impact evaluation of the development of electric vehicles on the power grid side in the plateau weak current network area.
\end{abstract}

Keywords: Tibet region; Distributed photovoltaic; Electric vehicle; Research application

\section{Introduction}

At present, when the global automobile industry is facing the great challenges of financial crisis and energy and environmental problems, a broad consensus has been formed in the world to develop electric vehicles, realize the electrification of automobile energy power system and promote the strategic transformation of traditional automobile industry. Especially in recent years, with the significant progress of high energy storage density and low-cost battery technology, the driving technology has become more and more mature and perfect, which promotes the performance of electric vehicles closer to fuel vehicles. At present, an upsurge of electric vehicle development has formed in the world[1].

All or part of the vehicles driven by motors and equipped with high-capacity electric energy storage devices are collectively referred to as electric vehicles. The components of electric vehicles include: electric drive and control system, driving force transmission and other mechanical systems, working devices to complete established tasks, etc. At present, there are three types of electric vehicles: pure electric vehicle, hybrid electric vehicle and fuel cell electric vehicle. Pure electric vehicle is the main development goal of all countries in the world. It is a vehicle powered by secondary batteries (such as lead-acid battery, nickel cadmium battery, nickel hydrogen battery or lithium-ion battery).

At present, China has formulated a unified standard for charging facilities, which compulsorily regulates the specification and size of charging interface and power supply. The power supply adopts $50 \mathrm{~Hz} 380 \mathrm{~V}$ and $220 \mathrm{~V}$ $\mathrm{AC}$ to obtain DC through rectification to charge the battery. However, the battery type, parameters and corresponding DC charging parameters have not been standardized. After consulting the literature, there is no report on photovoltaic charging products[2].

Electric vehicle is a vehicle with broad development prospects in China in the future. Electric vehicle charging station provides energy supply for the operation of electric vehicles, which is an important supporting infrastructure for the development of electric vehicles. At the same time, the development of electric vehicle charging station has also become a key problem restricting the wide promotion and use of electric vehicles. The electric vehicle charging station based on the large power grid should not only solve the impact of the instantaneous load on the power grid when the electric vehicle is rapidly charged, but also reduce the impact of harmonic pollution on power quality during charging. Therefore, the conventional power grid is difficult to meet and must be targeted[3]. This involves the transformation of the entire national power grid, which costs a lot. It can not be realized overnight from discussion, project approval to network completion. Therefore, photovoltaic power generation with the advantages of safety, energy conservation and environmental protection, simple maintenance and inexhaustible resources is used as the charging power supply of electric vehicles. The design and research of two-way energy storage photovoltaic grid connected electric vehicle charging system is carried out to meet the charging demand of electric vehicles without transforming the conventional power grid and affecting the power quality.

\footnotetext{
a 853969982@qq.com
} 


\section{Objectives of the project}

\subsection{Project requirements analysis}

Electric vehicles replace oil with electricity, which can achieve "zero emission" and low noise. It is an important means to solve energy and environmental problems. With the shortage of oil resources and the development of battery technology, electric vehicles have been close to or even better than traditional fuel vehicles in performance and economy, and began to be gradually popularized and applied all over the world. The new generation of energysaving and environment-friendly vehicles represented by electric vehicles is the inevitable trend of the development of automobile industry, which has become a general consensus. To promote electric vehicles, perfect supporting infrastructure is indispensable, and the construction of electric vehicle charging station is the key. The project will make use of the abundant solar energy resources in Lhasa and select the photovoltaic power generation system and energy storage system to form a two-way energy storage photovoltaic grid connected electric vehicle charging system. On the premise of not adding additional conventional power grid capacity, the impact of temporary rapid charging of electric vehicles on power grid load and the occurrence of load unevenness will be avoided through the buffer effect of energy storage unit, Eliminate the influence of harmonic pollution on power grid during charging[4].

The implementation of the project in this paper can not only solve the charging problem of electric vehicles in trial operation in Lhasa by using Lhasa's rich solar energy resources without increasing power grid capacity, investment and quantities, broaden the development and utilization channels of clean energy represented by solar energy resources, and achieve the purpose of energy conservation and emission reduction, Explore electric vehicle charging technology and electric vehicle detection and evaluation methods suitable for the actual situation of Tibet, make electric vehicles acceptable to the masses, accelerate the application and popularization of electric vehicles in our region, improve the professional skills of project participants through professional and technical personnel training, and reserve talents for the implementation of similar projects in the future.

\subsection{Difficulty analysis of the project}

(a) Complete the design and research of two-way energy storage photovoltaic grid connected electric vehicle charging system which can be connected to the grid and operate independently, and master the integration technology. Conventional photovoltaic power stations use low current and long-time discharge, while energy storage photovoltaic power stations used for electric vehicle charging are high current and short-time discharge. Special research needs to be carried out on the control system, the ratio of inverter device and storage bottle, and the battery management system (CMS) of such power stations. (b) Carry out the research and development of bidirectional converter and key technology of lead-acid battery energy storage system. At present, electric vehicle charging facilities are only applicable to AC, and there is no DC charging technology and equipment, which can be obtained only through special research and development. (c) Design and development of two DC / DC electric vehicle charging piles with fast charging, slow charging, multiple power levels and multiple output modes to meet the charging needs of different electric vehicles;

(d) Carry out power battery performance detection, evaluation and test research, and actually verify and test the actual adaptability of electric vehicles in plateau areas, so as to provide experimental data for electric vehicle manufacturers to improve and improve existing models to meet plateau applications;

(e) Explore the operation and management mode of electric vehicles and photovoltaic charging stations to provide reference for large-scale promotion and application in the future.

\section{Technology roadmap}

\subsection{Distributed photovoltaic system}

Electric vehicles are different from fuel vehicles. Because the voltage of power supply system and motor power system on the vehicle, whether AC or DC, is greater than the safety voltage, or even more than $380 \mathrm{~V}$, the detection of safety performance of electric vehicles is particularly important. It is before other performance tests and is the premise for the safe operation of electric vehicles. The test of electric energy consumption rate is an important special project of electric vehicles. How to test various electric parameters of electric vehicles, especially to obtain accurate real-time parameters under operation state, is the accurate basis for the study of electric energy consumption of electric vehicles. The energy consumption of pure electric vehicles is affected by many factors, including charger efficiency, control system energy consumption, motor efficiency and other energy consumption, which are the total energy consumption. It mainly tests the maximum speed, average speed, energy consumption, energy consumption per $100 \mathrm{~km}$, as well as the battery terminal voltage and output current, power consumption, feedback power, etc. during the operation of electric vehicles.

As shown in Figure 1, the principle of the two-way energy storage photovoltaic grid connected charging station is to store the electric energy generated by the photovoltaic power generation system and the electric energy obtained from the low point of the power grid with the battery, and charge the electric vehicle when needed. When there is no electric vehicle to be charged, when its own battery is full, the excess power is sent to the power grid to obtain additional income. The two-way energy storage photovoltaic grid connected charging station stores the electric energy obtained during its own power generation and the trough of the power grid, so it is not affected by power failure and cloudy and rainy weather; Through the buffer function of the energy storage system, the adverse 
impact on the power grid is avoided; Two way energy storage design enables the system to be connected to the grid or run independently.

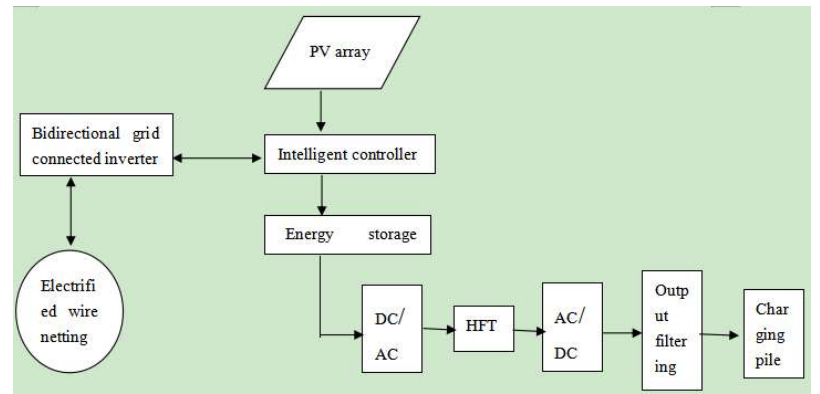

Fig. 1 Schematic diagram of bidirectional energy storage photovoltaic grid connected charging station

\subsection{Energy control system}

As shown in Figure 2, the power station judges and completes the automatic switching of power supply from PV array or power supply from power grid through intelligent controller, coordinates the output power of charging, battery energy storage and solar grid connected system, realizes the control and island detection of distributed power supply system, ensures safety and verifies the possibility of off grid operation. In case of power loss of the energy storage system, it is preferred to obtain electric energy from the whole PV train. When the PV array has no power at night or in cloudy and rainy weather, the power station obtains electric energy from the power grid through the bidirectional grid connected inverter according to the setting instruction, and then supplies power to the energy storage system through the intelligent controller. When the power of the energy storage system is sufficient, the PV array passes through the intelligent controller and supplies power to the power grid through the bidirectional grid connected inverter. The energy storage system is equivalent to UPS for the whole system. After passing through the energy storage system, all output electric energy of the charging station is boosted and filtered, and then transmitted to the charging pile. Energy flow of charging station circuit: Loop 1 realizes photovoltaic energy storage function: the electric energy emitted by solar cell modules is stored in the energy storage system, which is DC / DC conversion. As we all know, the electric energy emitted by the sun is unstable and changes with time, illumination and temperature, which requires a wide input range of the front stage DC, so as to make the most efficient use of solar energy. Loop 2 realizes the photovoltaic power generation function: the sunlight is sufficient, and the electric energy generated by the photovoltaic system fully meets the power supply demand of the charging station. At the same time, there is surplus. It can be considered to sell electricity to the power grid through this circuit to pursue economic benefits. Loop 3 realizes the power collection function of the energy storage system from the power grid: when the power generation of the PV array is insufficient, the system is controlled by the intelligent controller through the preset command, and takes power from the power grid through the bidirectional grid connected inverter to charge the energy storage system. Loop 4 realizes the power supply function of the charging station to the charging pile. When the charging pile works, the intelligent controller controls the energy storage system to output the DC power after boosting and filtering.

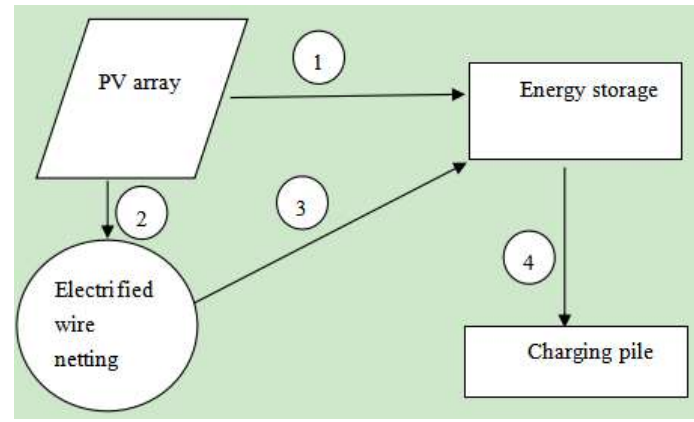

Fig.2 Block diagram of energy flow

For the bidirectional grid connected inverter, in order to realize the power exchange between the energy storage photovoltaic charging system and the grid connected power grid, the whole energy storage photovoltaic grid connected charging device can operate safely, stably and reliably. The bi-directional grid connected inverter will complete the research, design and development of the grid connected inverter based on the mode smooth switching technology based on droop characteristics, seamless switching of charging mode and grid connected and off grid charging control technology, and produce a $380 \mathrm{~V} /$ $20 \mathrm{~kW}$ bi-directional grid connected inverter prototype.

For the intelligent control system, in order to realize the management, monitoring and regulation of the whole charging station, the project team will design and develop an intelligent control system. The system will be responsible for monitoring the operation of the whole charging station system, completing the automatic switching between power supply of photovoltaic power generation system and power supply from the power grid, and coordinating the output power of charging, battery energy storage and solar grid connected system, It can realize the control and island detection of distributed power supply system, ensure safety and verify the possibility of off grid operation. A man-machine interface is established to control the switching management system of photovoltaic power generation and power grid power supply and the data acquisition and storage system of electric vehicle power battery charge according to the commands input in the man-machine interface.

For the charging pile, due to the use of the battery provided by the photovoltaic power station for charging, the inverter link and the resulting power loss are reduced, and the system is simpler than the AC charging pile. However, the electrical insulation strength and equipment heat dissipation level need to be increased. At the same time, in order to meet the different needs of various electric vehicles for charging parameters, the charging line is more inclusive, that is, the same line and its control unit can meet the requirements when the charging parameters are adjustable. 
The charging pile will be composed of man-machine exchange interface, monitoring system, display terminal, actuator, reserved terminal and terminal at the output end, or special charging gun and safety protection device connected to electric vehicle. The charging pile is equipped with a specific human-computer interaction interface to $\log$ in. Through the interface, corresponding charging operations such as charging mode and charging time setting can be carried out. The charging pile display screen can display data information such as charging amount and charging time, which can provide users with more power options and more output modes to meet the needs of users for fast charging and slow charging.

\section{Benefit analysis}

Through the implementation of the project in this paper, the demonstration and promotion of $18 \mathrm{~kW}$ charging station is calculated according to the average sunshine of 5 hours per day, the PV array power of the power station is $18 \mathrm{~kW}$, and the annual power generation is $18 \mathrm{~W} \times 5$ hours / day $\times 300$ days / year $\times 0.8$ (conversion efficiency) $=27000 \mathrm{~kW} \cdot \mathrm{h} /$ year. Calculated at 1 yuan per kilowatt hour, the direct economic benefit each year exceeds 27000 yuan; The completed charging station is expected to generate $27000 \mathrm{kWh}$ per year, which can also alleviate the local scarce power resources. Through the centralized application and promotion of photovoltaic grid connected power generation technology, the project will effectively promote the development of photovoltaic power generation technology and product market.

After the completion of the charging station, only the electric energy generated by the PV array can fully meet the driving needs of two small pure electric vehicles. The average daily driving mileage of each small electric vehicle is calculated as $50 \mathrm{~km}$. Compared with similar fuel powered vehicles, the fuel cost for fuel car driving $100 \mathrm{~km}$ is calculated as 80 yuan, The cost of pure electric vehicles per kilometer is only 1 / 8 of that of fuel powered vehicles, and each electric vehicle only saves about 10000 yuan of fuel cost per year.

\section{Conclusion}

Through the implementation of the project in this paper, it can be realized: (a) there is no need to expand the capacity of the existing power grid, which reduces the capital investment and engineering quantity; (b) When the electric energy generated by the photovoltaic power generation system is not enough, it can take electricity from the power grid at the low ebb of the power grid, which plays the role of peak cutting and valley filling, so as to make effective use of the excess electric energy at the low ebb of the power grid. (c) the electric energy stored in the battery can quickly charge the electric vehicle, which reduces the charging time, does not affect the power quality of the power grid and poses a threat to the power grid. (d) The energy storage photovoltaic grid connected charging system adopts modular design and simple capacity expansion. It only needs to increase the battery pack without reconstruction. (e) The two-way energy storage design enables the charging station to complete both grid connection and independent operation. After the power grid outage, the charging station can also serve as a backup power supply system to temporarily supply power to local buildings.

\section{References}

1. Zahedi A. Maximizing solar PV energy penetration using energy storage technology[J]. Renewable and Sustainable Energy Reviews, 2011, 15 (1) : 866-870.

2. García-Villalobos J, Zamora I, San Martín J I, et al. Plug-in electric vehicles in electric distribution networks: A review of smart charging ap- proaches [J . Renewable and Sustainable Energy Reviews, 2014, 38: 717-731.

3. Zhao J, Wang C, Zhao B, et al. A Review of Active Management for Distri- bution Networks: Current Status and Future Development Trends[J]. Elec- tric Power Components and Systems, 2014, 42(3-4) :280293.

4. Cheng L, Chang Y, Huang R. Mitigating Voltage Problem in Distribution System With Distributed Solar Generation Using Electric Vehicles[J]. IEEE Transactions on Sustainable Energy, 2017, 6( 4) : 1475-1484. 